\title{
NON-DESTRUCTIVE ASSESSMENT OF THE ADHESION AT THE INTERFACE BETWEEN FRCM REINFORCEMENTS AND MASONRY SUBSTRATES BY NON-LINEAR ULTRASONIC TECHNIQUE
}

\section{ANNA CASTELLANO ${ }^{1 *}$, AGUINALDO FRADDOSIO ${ }^{2}$, TRIBIKRAM KUNDU $^{3}$ AND MARIO DANIELE PICCIONI ${ }^{2}$}

\author{
${ }^{1}$ Department of Mechanics, Mathematics and Management (DMMM) \\ Polytechnic University of Bari \\ via Re David, 70125 Bari, Italy \\ e-mail: anna.castellano@poliba.it (*corresponding author) \\ ${ }^{2}$ Department of Civil Engineering Sciences and Architecture (DICAR) \\ Polytechnic University of Bari \\ via Re David, 70125 Bari, Italy \\ e-mail: aguinaldo.fraddosio@poliba.it,mariodaniele.piccioni@poliba.it \\ ${ }^{3}$ Department of Civil \& Architectural Engineering \& Mechanics \\ The University of Arizona \\ 1209 E. Second St., Tucson, AZ 85721, USA \\ Email: tkundu@email.arizona.edu
}

Keywords: Masonry, FRCM Reinforcements, Non-Destructive Tests, Nonlinear Ultrasonic, SPC Technique.

Abstract. The strong diffusion of Fiber Reinforced Cementitious Matrix (FRCM) composites for the reinforcement of historic masonry constructions requires suitable experimental techniques for assessing possible defects in the adhesion between FRCM and masonry, and between FRCM layers. To this aim, in this paper, an innovative nonlinear ultrasonic approach based on the Side-band Peak Count (SPC) technique is proposed. This approach is discussed and validated through experimental tests made on tuff substrates reinforced with FRCM mortars embedding a basalt fibers grid and having known artificial defects at the adhesion between tuff and FRCM as well as in the fiber grid.

\section{INTRODUCTION}

In recent years, the development of suitable strengthening techniques for historic masonry constructions has received increasing attention from research community. One of the main motivations is the pronounced seismic vulnerability of the built heritage made of masonry. Among possible strengthening materials, Fiber Reinforced Cementitious Matrix (FRCM) composites have gained interest over the classical Fiber Reinforced Polymer (FRP) composites since FRCM composites appear to be more compatible with historical masonries, both from the technological and the structural point of view ${ }^{[1]}$. This is mainly due to the fact that FRCM composites are based on a mortar matrix embedding high-strength long fiber grids (Glass, Carbon, Basalt, etc.); as well as matrix containing short reinforcement fibers. It should 
be noted that the relatively low cost of FRCM reinforcements makes it possible to build distributed reinforcements, that reveal to be much more effective than the localized ones (like those built by FRP strips) in view of improving the overall structural performances of masonry constructions.

A number of studies concern the interactions between FRCM composites and masonry, the adhesion and the delamination of FRCM reinforcement from masonry supports, investigating possible collapse modes and the overall structural capacity of the strengthened constructions, both from the theoretical and the experimental point of view ${ }^{[2-3]}$. Anyway, the problem of the detection of possible defects in the adhesion between FRCM and masonry and of the fiber grids, formed during the application of the reinforcement or during the service life of the construction, for example due to exceptional loads like earthquake actions, fire, etc., needs to be further investigated. Indeed, these defects may strongly undermine the strength of the reinforced construction. Thus, suitable experimental techniques must be developed.

On this issue the literature is rather limited, even if the field of application is widened to include also the FRP reinforcements ${ }^{[4]}$. Here, an innovative non-linear ultrasonic technique is proposed for detecting defects in the adhesion between FRCM composite layers and masonry substrates.

Non-linear ultrasonic techniques may be very effective for detection of defects and damage [5]. Here the principle that is exploited for experimental evaluations is that defects act like an active non-linear radiation source of new frequency components rather than passive scatters as in the case of classical linear ultrasonic techniques [6]. Based on this, the Side-band Peak Count (SPC) technique level of the non-linearits additional minor compo Experiments perforn ity of
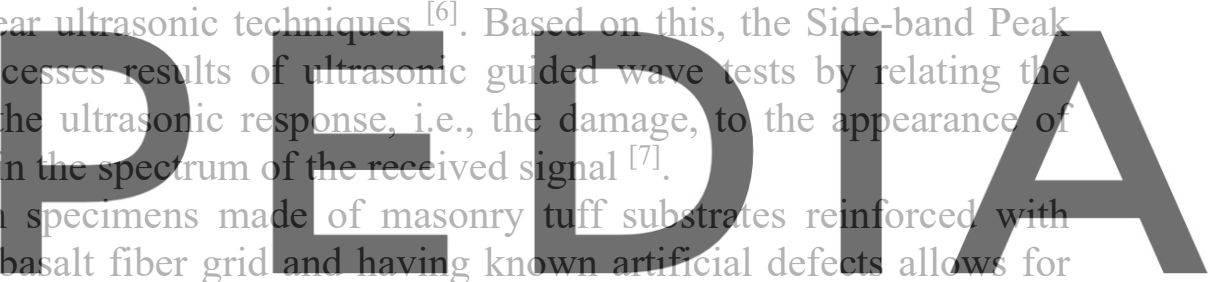

discussing the proposed approach. In particular, those defects represent a small and a large

Register fojsfyeenat the fiber net. Experimental results show the effectiveness of SPC technique in detecting the defects and allows for highlighting the influence of the defect dimensions on the variation in SPC.

\section{NON-LINEAR ULTRASONICS AND SPC TECHNIQUE}

Acoustic (sonic and ultrasonic) Non-Destructive Testing techniques represent one of the most important NDT approach for testing material and structures. The difference between sonic and ultrasonic tests relies on the frequency of the acoustic waves employed in the experiments, below $20 \mathrm{kHz}$ for sonic tests, and above that frequency threshold for ultrasonic tests. Sonic tests are suitable for very dispersive and/or very thick specimens but are characterized by poor accuracy in the detection and localization of defects. On the other hand, ultrasonic tests allow for a definitely better resolution and accuracy, but generally their application to masonry constructions is strongly obstructed by the high attenuation of ultrasonic waves ${ }^{[8]}$.

Here the attention is given on ultrasonic tests, since the propagation material is represented by FRCM layers, where the attenuation is not such that it would prevent the acquisition of 
signals with enough energy.

The traditional approach for ultrasonic tests, i.e., the classical linear ultrasonic approach, is based on the analysis of so-called linear acoustic parameters like flight time (and, consequently, wave speed), resonance frequency and attenuation of acoustic waves. Ultrasonic waves are reflected and scattered by internal cracks; these phenomena can be analyzed from the linear analysis of the interaction between the material defects and the propagating elastic waves. Indeed, internal damage can be detected and characterized based on the study of velocity and attenuation of elastic waves in the material.

However, the wave propagation-based linear ultrasonic techniques fail to detect small defects, much smaller than the wavelength of the interrogating ultrasonic signals, or particular kind of defects like those represented by localized discontinuities in the adhesion between layers of multilayer materials.

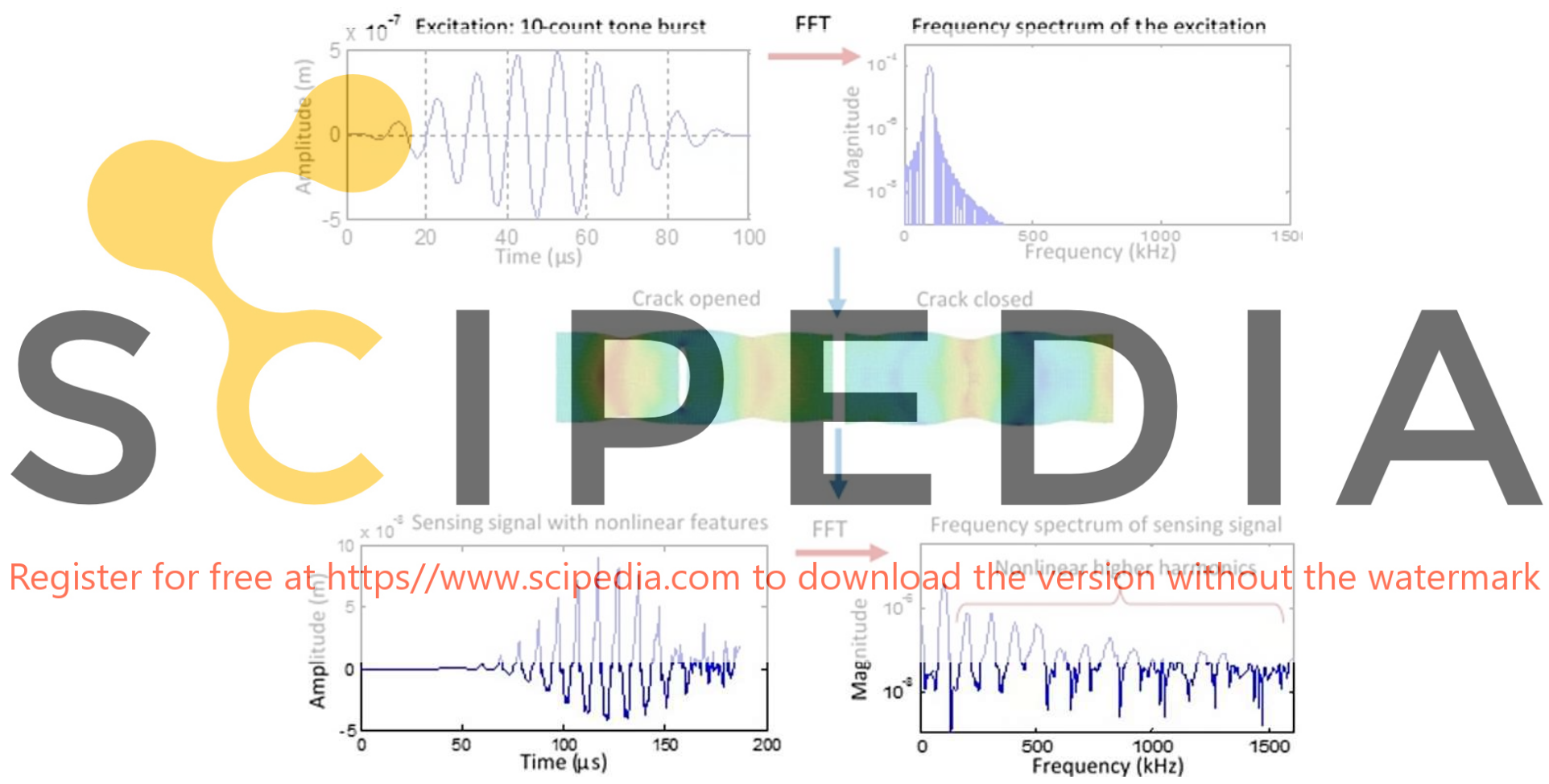

Figure 1: Generation of higher harmonics due to the damage [9]

In order to overcome the above limitations, in recent years a lot of research works appeared in the literature about non-linear acoustical/ultrasonic techniques. Nonlinear acoustical techniques are based on nonlinear stress-strain relationship assumptions. A very important aspect is that when two waves of different frequencies propagate through a linear material their frequencies do not change. But, if the material is non-linear, then the interaction between these two waves produces secondary waves having frequencies that are linear combinations of the frequencies of the primary waves. Even when a wave of single frequency propagates through a non-linear material new waves of higher frequency (higher harmonics) and lower frequency (subharmonics) can be generated in the material. Figure 1 shows that if a signal is 
sent through a nonlinear material (in particular, due to an internal crack defect), in addition to the fundamental frequency the higher frequencies (higher harmonics) - multiples of the fundamental frequency - appear in the spectrum of the received signal.

Moreover, in a nonlinear system both resonance frequency and attenuation coefficient change with the vibration amplitude.

Higher harmonic, subharmonic, and modulated waves can be effectively used for detecting defects. Various non-linear ultrasonic techniques have been developed for internal defect detection by efficiently using higher harmonic, subharmonic, and modulated waves. Indeed, even tiny discontinuities or defects can produce detectable acoustic nonlinearity; the detectability comes from the fact that defect-related nonlinearity is orders of magnitude higher than the intrinsic nonlinearity of the intact material. Thus, nonlinear ultrasonics is very sensitive to small defects like fatigue cracks, fiber debonding and delamination, and is very interesting because the onset of the damage can be assessed in a very early stage.

Here, an advanced nonlinear ultrasonic technique is studied with reference to the analysis of adhesion defects and delamination of FRCM reinforcements of masonry structures: the Sideband Peak Count (SPC) technique which was proposed first in ${ }^{[10]}$ for the analysis of prismatic bars. SPC is based on the nonlinear interaction between different Lamb wave modes: if the Lamb wave is generated by a broadband excitation then multiple Lamb modes are generated and, if the plate material is nonlinear (this can be, for example, due to the damage), then the interaction between these various Lamb modes can generate multiple

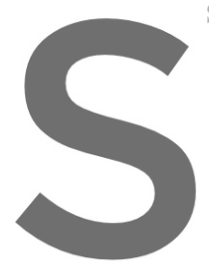
sidebands (see Fig. 2)
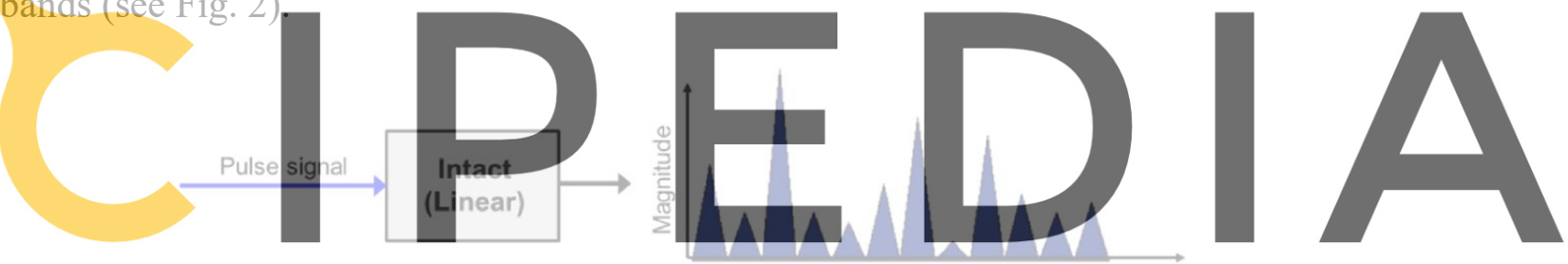

Register for free at https//www.scipedia.com to download the version without the watermark

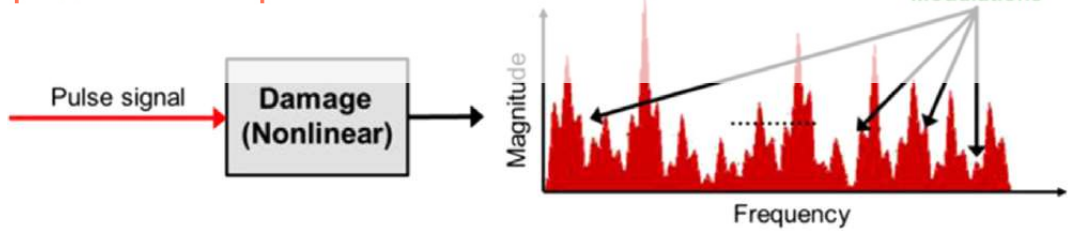

Figure 2: Generation of sideband peak due to the wave propagation through a damaged (nonlinear) medium ${ }^{[11]}$

In particular, the SPC technique is a feature extraction technique aimed at quantifying the degree of nonlinearity in a damaged structure from its response to broadband pulse excitations. The level of nonlinearity, related to the damage, is associated with the number of peaks of the sidebands; in particular, the number of peaks above a suitably taken threshold value is counted (see Fig. 3-a). It is also possible to gather information on the damage by comparing the number of peaks of the sidebands above a threshold value for the initial (undamaged) state and the current (damaged, nonlinear) state, i.e., by studying the SPC difference: Figure 3-b highlights that the damaged specimen shows a significantly higher number of frequency-domain peaks especially for low values of the threshold. 
For the representation of SPC results, usually diagrams of the SPC parameter (1), defined as the ratio of the number of sideband (modulation) peaks $\mathrm{N}_{\text {peak }}$ over a moving threshold (th) to the total peak number $\mathrm{N}_{\text {tot }}$ in the considered frequency domain, versus the amplitude of the threshold are employed.

$$
S P C(t h)=\frac{N_{p e a k}(t h)}{N_{\text {tot }}}
$$

a

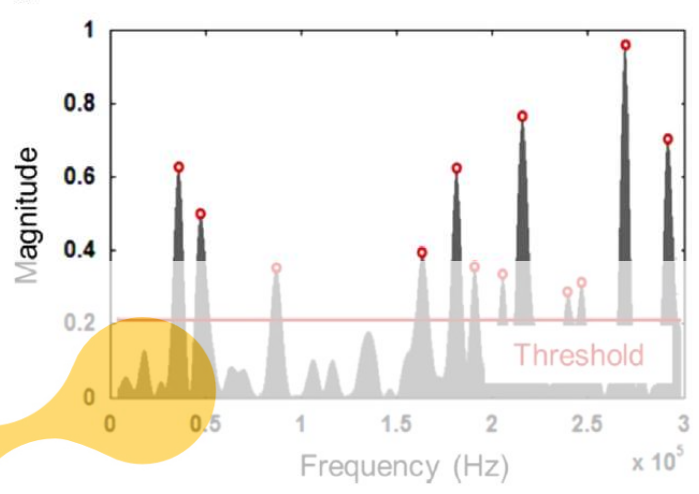

b

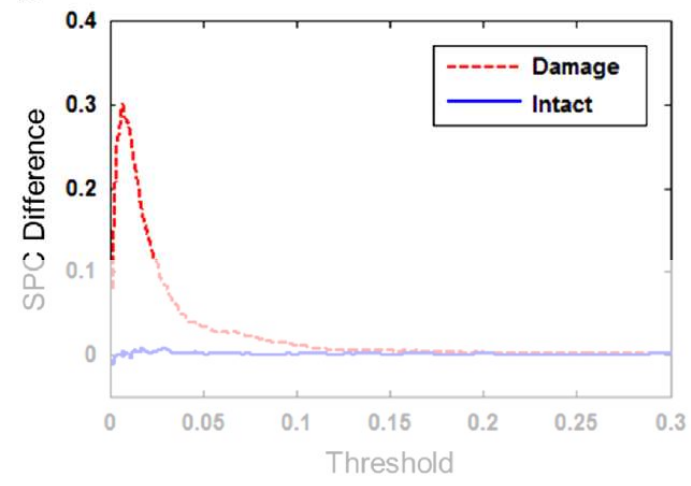

Figure 3: Illustration of the Sideband Peak Count (SPC) technique ${ }^{[1]}$

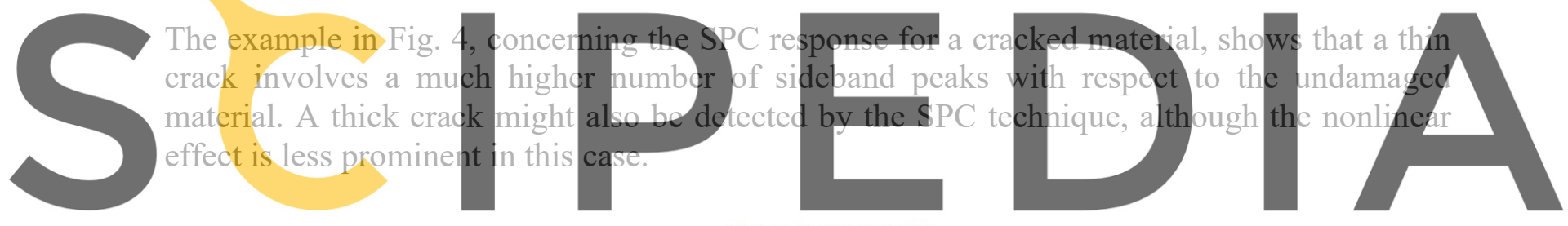

Threshold Region [0-0.2]

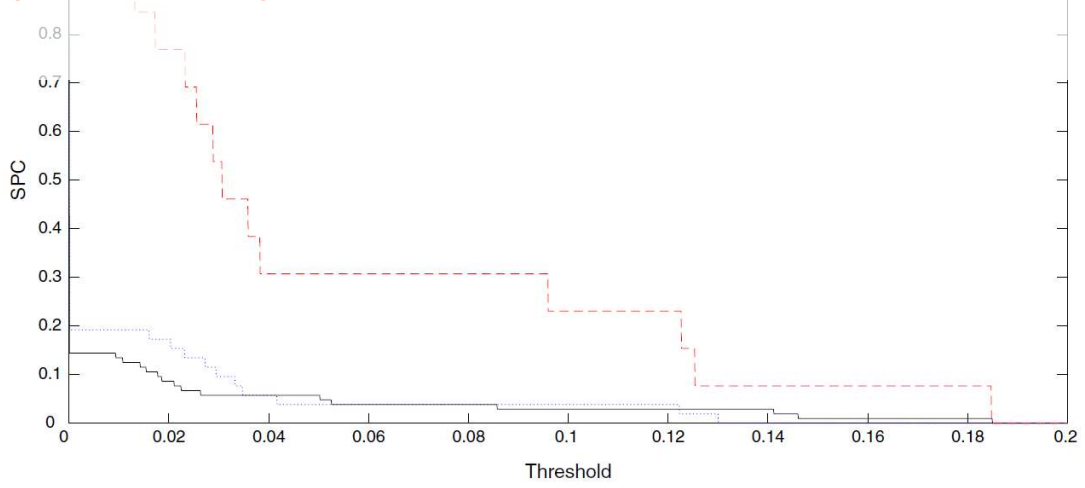

Figure 4: SPC response in case of thin or thick cracks ${ }^{[12]}$

SPC has been successfully applied in fields like inspection of concrete ageing, monitoring of heat treatments of metals, detection of fatigue damage in metals, etc. ${ }^{[13]}$. It should be emphasized that, although the data processing needed for SPC is different from that of classical linear ultrasonic methods, the experiments can be performed by standard ultrasonic 
devices. Thus, SPC appears to be very interesting in view of practical applications, especially in fields where only the use of simple instruments is allowed such as for monitoring the strengthening of masonry constructions.

\section{EXPERIMENTAL TESTS}

The purpose of the experimental tests performed at "Laboratorio Ufficiale Prove Materiali M. Salvati" of Polytechnic University of Bari is to study the capability of SPC nonlinear ultrasonic technique for characterizing defects in FRCM reinforcements of masonry constructions.

To this aim, 5 pairs of specimens consisting in a tuff support with a FRCM reinforcement embedding a basalt fiber grid have been analyzed: 2 of them (labeled S1 and S2) are undamaged; 2 specimens (labeled SDIA1 and SDIA2) present a discontinuity in the adhesion of the FRCM layer obtained by interposing between tuff and FRCM a $20 \mathrm{~mm}$ wide scotch tape (Fig. 5-a, above); 2 specimens (labeled SDIB1 and SDIB2) are similar to the previous pair, except for the width of the discontinuity in the adhesion, obtained by a $30 \mathrm{~mm}$ wide scotch tape (Fig. 5-a, below); for 2 specimens (labeled SDFA1 and SDFA2) a defect in the fiber-mortar matrix interface is simulated by a small cut about $15 \mathrm{~mm}$ wide in the basalt fiber grid (Fig. 5-b, above); finally, for 2 specimens (labeled SDFB1 and SDFB2) a defect in the fiber-mortar matrix interface is simulated by a large cut about $30 \mathrm{~mm}$ wide in the basalt fiber grid (Fig. 5-b, below).
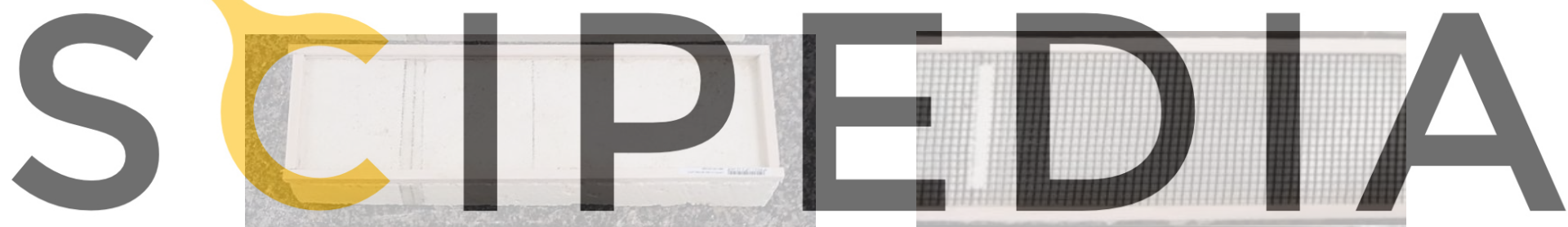

Register for free at https//www.scipedia.com to download the version without the watermark
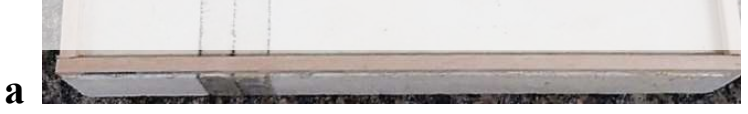

b

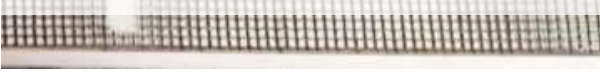

Figure 5: Artificial damage in the specimens: a) small $(20 \mathrm{~mm})$ and large $(30 \mathrm{~mm})$ defect of FRCM-tuff adhesion; b) small $(15 \mathrm{~mm})$ and large $(30 \mathrm{~mm})$ defect in the grid.

All specimens are prismatic, with dimension of the face to be reinforced by the FRCM layer about $485 \mathrm{~mm} \times 150 \mathrm{~mm}$; the overall thickness of the FRCM layer is $10 \mathrm{~mm}$. The defects are located about $110 \div 125 \mathrm{~mm}$ from one of the short edges of the face. FRCM reinforcement is made by a two-component, pre-blended, fiber-reinforced mortar embedding a basalt fiber grid $\left(6 \mathrm{~mm} \times 6 \mathrm{~mm}, 250 \mathrm{~g} / \mathrm{m}^{2}\right)$.

The ultrasonic setup is composed of an Olympus 5072PR ultrasonic pulser with two probes for longitudinal waves having a central frequency of $50 \mathrm{KHz}$; the emitting probe is inclined at 65 degree for generating surface waves in the FRCM layer. The signals are managed by an Agilent MSO-X-4954 oscilloscope.

For determining the ultrasonic response in the damaged area, the emitting probe has been 
kept fixed left to the defect, whereas the receiving probe has been placed on the defect (position 1) or on the right of the defect (position 2). For determining the reference ultrasonic response in the undamaged area, the two transducers have been placed on the right end of the specimen at the same distances of position 1 and position 2 (Fig. 6).
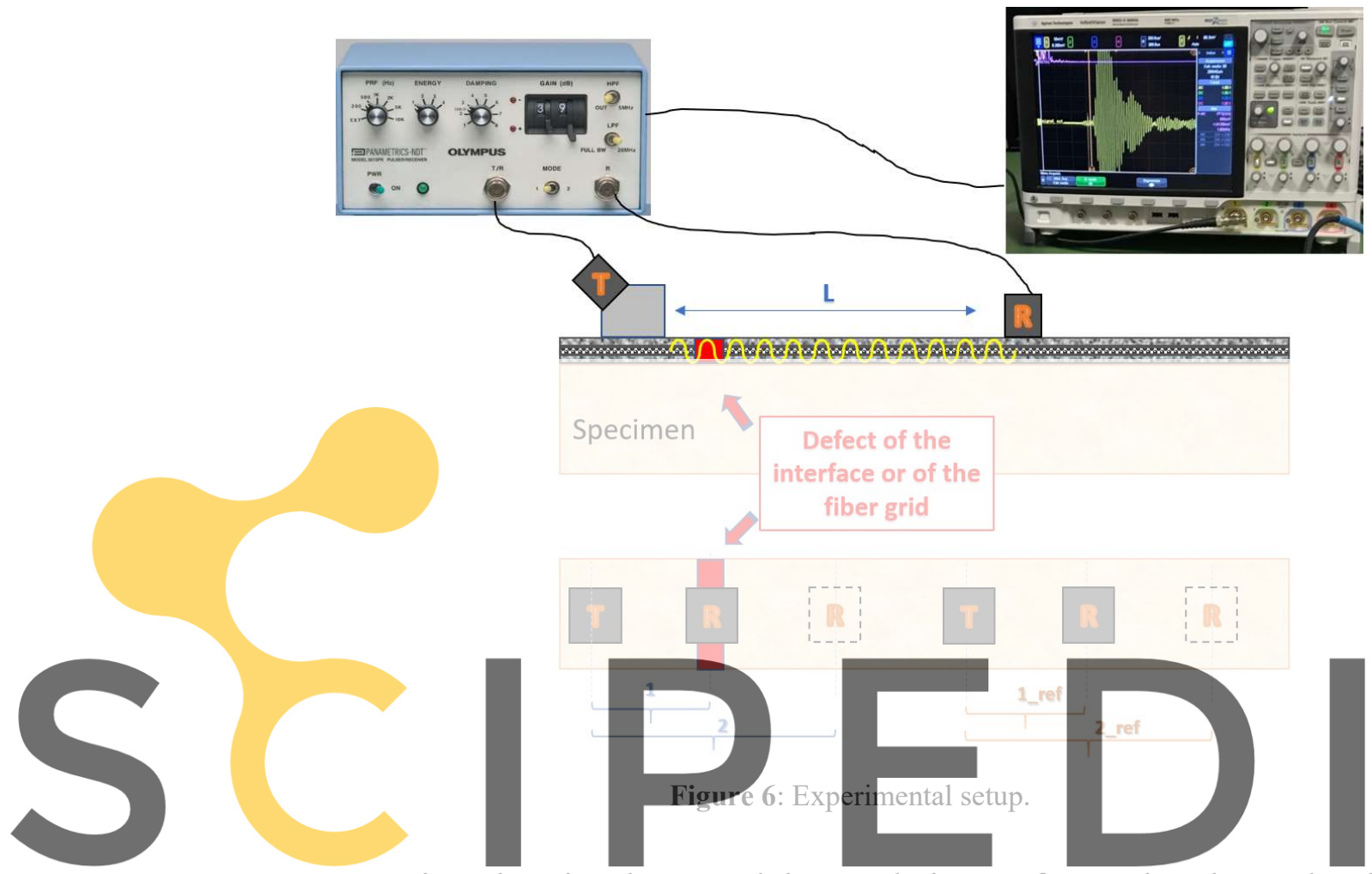

For reprocessing the signals, an ad hoc Labview software has been developed; this

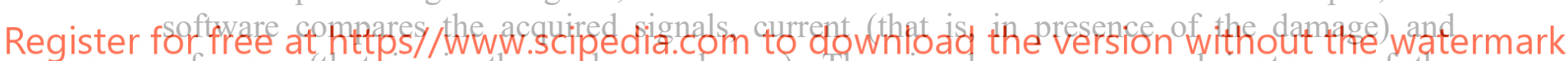
reference (that is, in the undamaged area). The signals are compared in terms of the waveform, of normalized frequency response function and of frequency spectra. Finally, the software performs the SPC evaluation of the acquired signals.

\section{RESULTS AND DISCUSSION}

In what follows, the diagrams of SPC results for the 8 artificially damaged specimens are shown and discussed. For the sake of brevity, all diagrams refer only to the case of receiving probe located in "position 2", i.e., on the right of the defect (receiving probe far from the defect). Moreover, only the results of one of the two specimens for each kind of defect is shown (the results for the specimens labeled "1", SDIA1, SDIB1, and so on). The influence of the defect on the SPC response can be inferred from the comparison between the SPC diagram obtained for signals acquired in the undamaged area (reference line, in orange) and the SPC diagram obtained for signals acquired in the damaged area (in light blue).

In Figure 7, the SPC response of the specimen with small adhesion defect SDIA1 is presented. In this case, the light blue curve, representing the SPC parameter for the damaged area is distinctly lower than the reference orange curve, representing the SPC parameter for signals acquired with the same distances between probes in the undamaged area, for threshold 
values above 0.01 . The different behavior below the very small threshold value of 0.01 is likely ascribable to noise in the signals and therefore it is not to be considered representative of difference due to the damage.

Figure 8 is analogues to Figure 7, but for the specimen with large adhesion defect SDIB1. Here, the curves are inverted with respect to those in Figure 7, with the light blue curve, representing the SPC parameter for the damaged area higher than the reference orange curve referring to the undamaged area.

SDIA1 [SPC: 0-0.2]

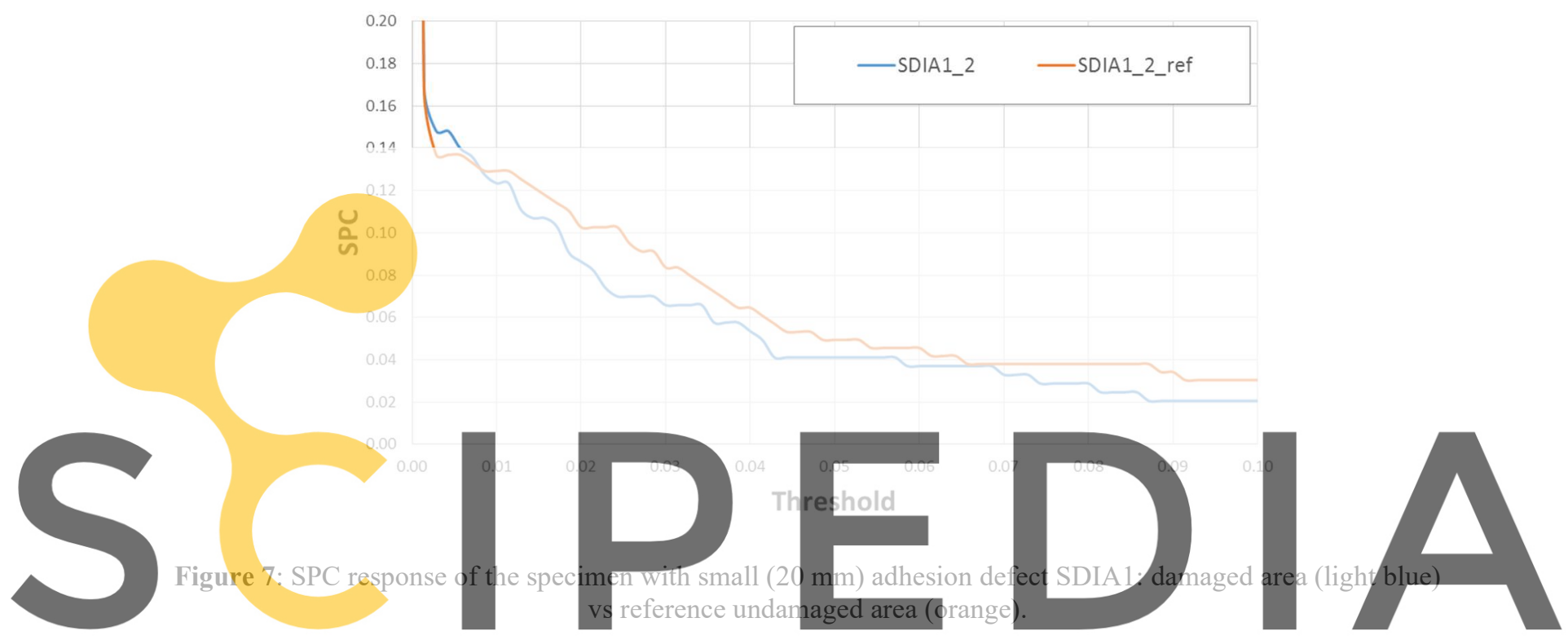

Register for free at https//www.scipedia.som1to downlgad the version without the watermark

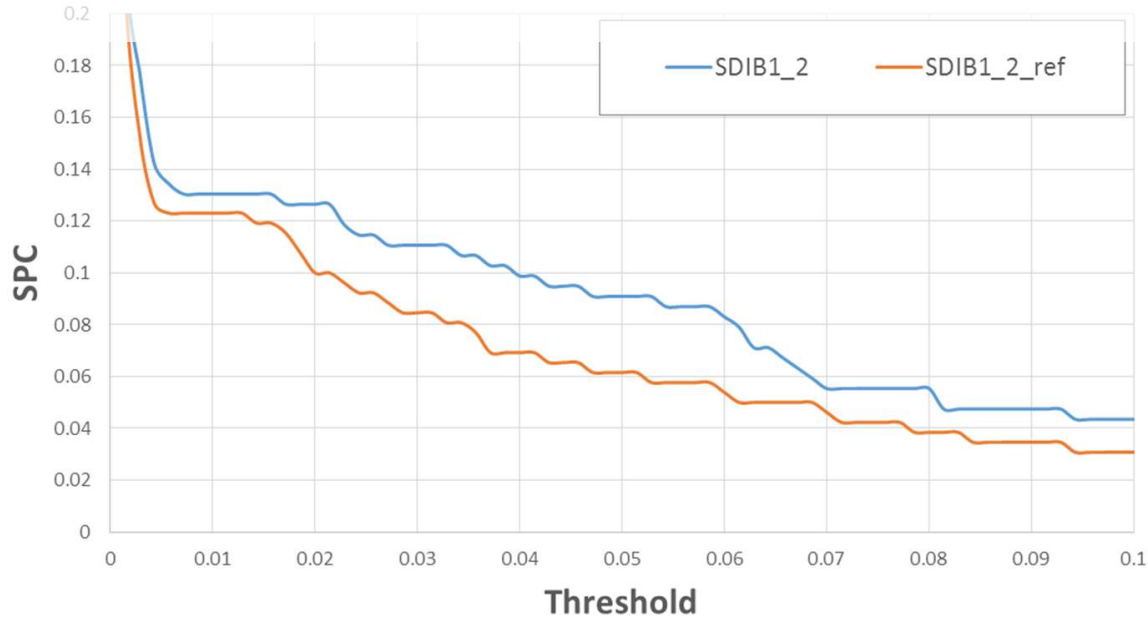

Figure 8: SPC response of the specimen with large $(30 \mathrm{~mm})$ adhesion defect SDIB1: damaged area (light blue) vs reference undamaged area (orange). 
In Figure 9, the SPC response of the specimen with small grid defect SDFA1 is presented. Also in this case, like for the case of the specimen with small adhesion defect SDIA1, the light blue curve, representing the SPC parameter for the damaged area is considerably lower than the reference orange curve, representing the SPC parameter for signals acquired for the same distances between probes, but in the undamaged area.

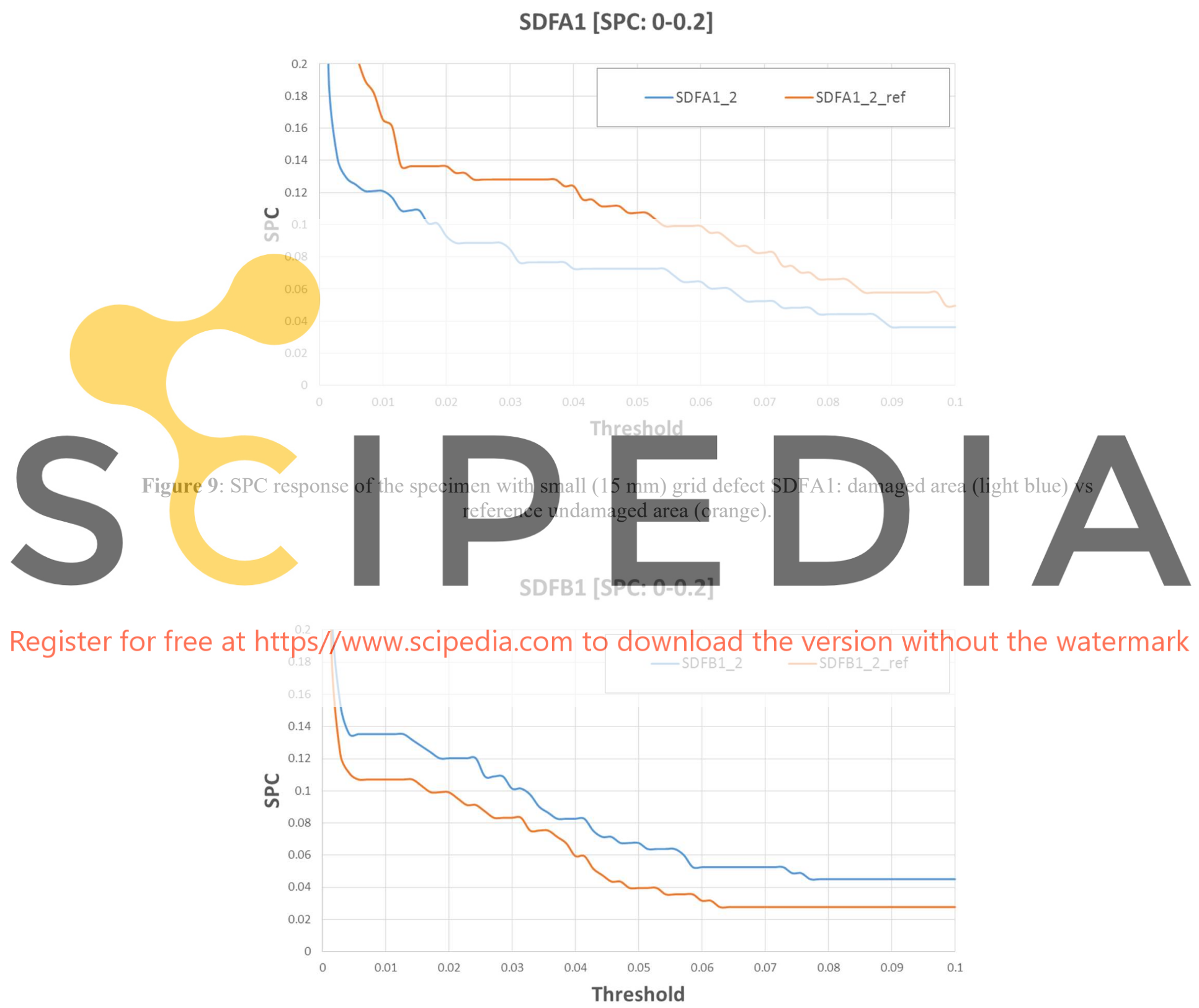

Figure 10: SPC response of the specimen with large $(30 \mathrm{~mm})$ grid defect SDFB1: damaged area (light blue) vs reference undamaged area (orange).

Finally, Figure 10 is analogues to Figure 9, but for the specimen with large grid defect 
SDFB1. Also in this case, like the specimen with large grid defect SDIB1, an opposite behavior with respect to those in Figure 9 is observed: the damage results show much higher values of the SPC (light blue curve) in comparison to the undamaged area (orange curve).

From the above results it is clear that SPC technique can identify even small defects of FRCM reinforcement. Indeed, for each examined specimen, embedding different kinds of artificial defects, a very different behavior between the damaged and the undamaged area in terms of SPC parameter values has been observed. Note that this conclusion holds also for the specimens labeled "2" (SDIA2, SDIB2 and so on), and for different positions of the receiving probe (the position called "position 1" in the above).

The SPC response of the specimens with large defects (adhesion defects, SDIB1 and SDIB2, and grid discontinuity, SDFB1 and SDFB2) is what is expected based on the literature: the defect involves a higher level of nonlinearity, and thus an increase of the SPC parameter values for all the threshold values considered. On the contrary, the SPC response of the specimens with small defects (adhesion defects, SDIA1 and SDIA2, and grid discontinuity, SDFA1 and SDFA2) is to some extent unexpected: the SPC curves of the undamaged area are systematically above the SPC curves of the damaged area. Notice that the experimental tests have been repeated multiple times in order of ascertain that the latter unexpected behavior was not caused by experimental errors.

Maybe a possible reason of this peculiar behavior relies on the wavelength of the employed ultrasonic waves and on the features of the examined media, composed by a relatively thin FRCM layer in adhesion on a tuff masonry substrate. Moreover, the ERCM layer itself can be subdivided in three sublayers, that is tho mo
embedding the fiber grid.
Indeed, the reference literature is mostly
whereas in the present case the tests involve
layer FRCM reinforcement. The examined
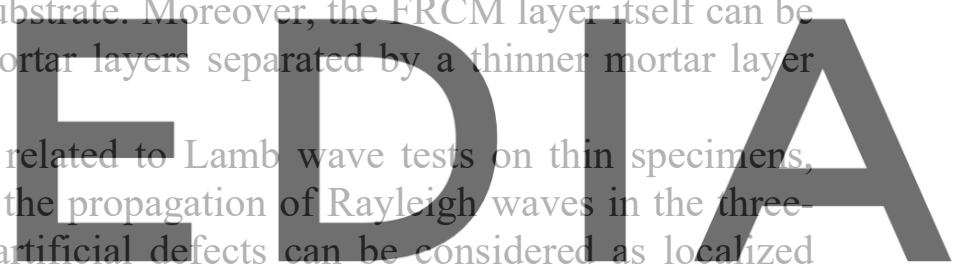

discontinuity conditions between layers in adhesion, that is, imperfection of the boundary

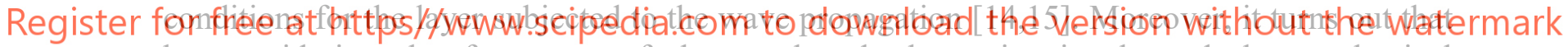
by considering the frequency of the employed ultrasonic signals and the mechanical parameters of the FRCM mortar, the ultrasonic wavelength have the same order of magnitude of the dimensions of the defects. Finally, a fact not irrelevant should be that the material being investigated is markedly nonlinear also in the undamaged state.

The above considerations open an intriguing and challenging research perspective in order to establish the reasons of the observed SPC behavior: to this aim suitable theoretical and numerical investigations have to be developed.

\section{CONCLUSIONS}

- $\quad$ The reported experiments on tuff samples reinforced by FRCM embedding basalt grids show that SPC nonlinear ultrasonic technique is capable of detecting defects in adhesion and in the fiber net, with quite different values of the SPC parameter in the damaged area with respect to those related to the undamaged area.

- This result appears interesting in view of applications mainly for three reasons. First, the proposed technique is simple to be applied, since standard ultrasonic equipment can be used: moreover, the developed Labview software is very fast in giving final 
results in terms of SPC curves. Second, nonlinear ultrasonic techniques like SPC technique allows for detecting defects also quite far from the application areas of the probes; sometimes nonlinear effects increase with the distance, whereas linear effects quickly decay. Third, SPC technique is capable of detecting small defects, out of reach of conventional linear ultrasonic techniques, and assessing the onset of the damage in its early stage.

- Finally, the obtained results encourage further theoretical and numerical analysis aimed at justifying the very different behavior observed for small and large defects.

\section{REFERENCES}

[1] Carozzi, F.G., Milani, G. and Poggi, C. Mechanical properties and numerical modeling of Fabric Reinforced Cementitious Matrix (FRCM) systems for strengthening of masonry structures. Compos. Struct. (2014) 107:711-725.

[2] Bove, M., Castellano, A., Fraddosio, A., Scacco, J., Milani, G. and Piccioni, M.D. Experimental and numerical analysis of FRCM strengthened parabolic tuff barrel vault. Key Eng. Mater. (2019) 817 KEM:213-220.

[3] Olivito, R.S., Codispoti, R. and Cevallos, O.A. Bond behavior of Flax-FRCM and PBO-FRCM composites applied on clay bricks: Experimental and theoretical study. Compos. Struct. (2016) 146:221-31.

[4] Büyüköztürk, O., Haupt, R., Tuakta, C. and Chen, J. Remote Detection of Debonding in FRPstrengthened Concrete Structures Using Acoustic-Laser Technique. In: O. Büyüköztürk et al. (Eds.): Nondestructive Testing of Materials and Structures, RILEM Bookseries 6 (2013), pp. 1924.

[5] Kundu, T. Ultrasonic nondestructive evaluation: Engineering and biological material characterization. CRC Press, (2003).

[6] Yan, D., Drinkwater, B.W., Neild, S.A. Measurement of the ultrasonic nonlinearity of kissing bonds in adhesive joints". NDT E Int. (2009) 42(5):459-466.

[7] Liu, P., Sohn, H., Kundu, T., Yang, S. Noncontact detection of fatigue cracks by laser nonlinear wave modulation spectroscopy (LNWMS)". NDT E Int. (2014) 66:106-116.

[8] Camassa, D., Castellano, A., Fraddosio, A., Piccioni, M.D. Improvements of the Ultrasonic Tomography for Applications to Historical Masonry Constructions. In: R. Aguilar et al. (Eds.): Structural Analysis of Historical Constructions, RILEM Bookseries 18 (2019), pp. 447-455.

[9] Shen, Y. and Cesnik, C.E.S. Nonlinear scattering and mode conversion of Lamb waves at breathing cracks: An efficient numerical approach. Ultrasonics (2019) 94:202-217.

[10] Eiras, J.N., Kundu, T., Bonilla, M. and Payá, J. Nondestructive monitoring of ageing of alkali resistant glass fiber reinforced cement (GRC). J. Nondestruct. Eval. (2013) 32(3):300-314.

[11] Liu, P., Sohn, H., Kundu, T. and Yang, S. Noncontact detection of fatigue cracks by laser nonlinear wave modulation spectroscopy (LNWMS). NDT E Int. (2014) 66:106-116.

[12] Hafezi, M.H., Alebrahim R. and Kundu T. Peri-ultrasound for modeling linear and nonlinear ultrasonic response". Ultrasonics (2017) 80:47-57.

[13] Kundu, T., Eiras, J.N., Li, W., Liu, P., Sohn, H. and Payá J. Fundamentals of Nonlinear 
Acoustical Techniques and Sideband Peak Count. In: T. Kundu (Ed.): Nonlinear Ultrasonic and Vibro-Acoustical Techniques for Nondestructive Evaluation, Springer, Cham (2019), pp. 1-88.

[14] Castellano, A., Foti, P., Fraddosio, A., Marzano, S. and Piccioni, M.D. Ultrasonic immersion tests for mechanical characterization of multilayered anisotropic materials. In EESMS 2014 2014 IEEE Workshop on Environmental, Energy and Structural Monitoring Systems, Proceedings (2014), pp. 63-68.

[15] Castellano, A., Fraddosio, A. and Piccioni, M.D. Quantitative analysis of QSI and LVI damage in GFRP unidirectional composite laminates by a new ultrasonic approach. Compos. B. Eng. (2018) 151: 106-117. 